\title{
NIF HEPW 2D Radiography Option Survey
}

Jeffrey A. Koch

April 28, 2004

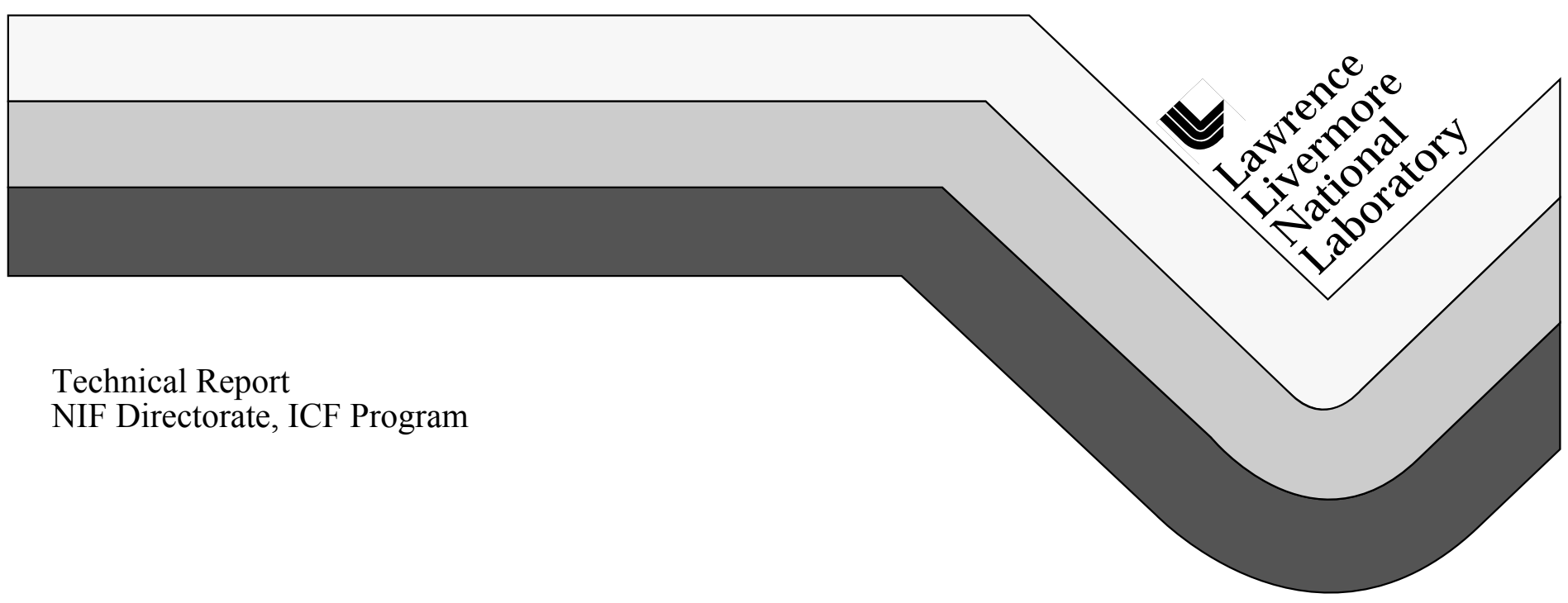




\section{DISCLAIMER}

This document was prepared as an account of work sponsored by an agency of the United States Government Neither the United States Government nor the University of California nor any of their employees, makes any warranty, express or implied, or assumes any legal liability or responsibility for the accuracy, completeness, or usefulness of any information, apparatus, product, or process disclosed, or represents that its use would not infringe privately owned rights. Reference herein to any specific commercial product, process, or service by trade name, trademark, manufacturer, or otherwise, does not necessarily constitute or imply its endorsement, recommendation, or favoring by the United States Government or the University of California. The views and opinions of authors expressed herein do not necessarily state or reflect those of the United States Government or the University of California, and shall not be used for advertising or product endorsement purposes. 
April 28, 2004

To: Distribution

From: Jeffrey Koch, L-481

Subject: NIF HEPW 2D Radiography Option Survey

UCRL-TR-203996

\section{Introduction}

Radiography experiments planned for the high-energy petawatt laser on the NIF have specific requirements for spatial resolution, field of view, x-ray energy, and signal-to-noise ratio. Based on existing data from smaller laser facilities, we can scale the source parameters up to the anticipated NIF laser parameters and quantitatively determine how the experimental requirements might be met. In this memo I investigate one specific materials experiment, and explore various options for meeting the experimental requirements with the expected NIF x-ray source based on an analysis of photon statistics.

\section{Statement of the Experiment}

The goal of the NIF experiment is to radiograph a laser-driven tantalum foil with thickness modulations. The required spatial resolution is $10 \mu \mathrm{m}$, and the required field of view is $1 \mathrm{~mm}$. The experiment must be able to measure foil thickness variations of $10 \%$ with an uncertainty of $10 \%$, i.e. with an uncertainty that is $1 \%$ of the thickness. Based on the target parameters, the required $\mathrm{x}$-ray energy is approximately $40 \mathrm{keV}$; quasimonochromatic $\mathrm{x}$-rays are desirable but not necessarily required provided the other experimental requirements can be met.

\section{Concept \#1}

Here we imagine simple point-projection backlighting, with the source monochromatized by one or more flat multilayer mirrors placed somewhere between the source and the detector.

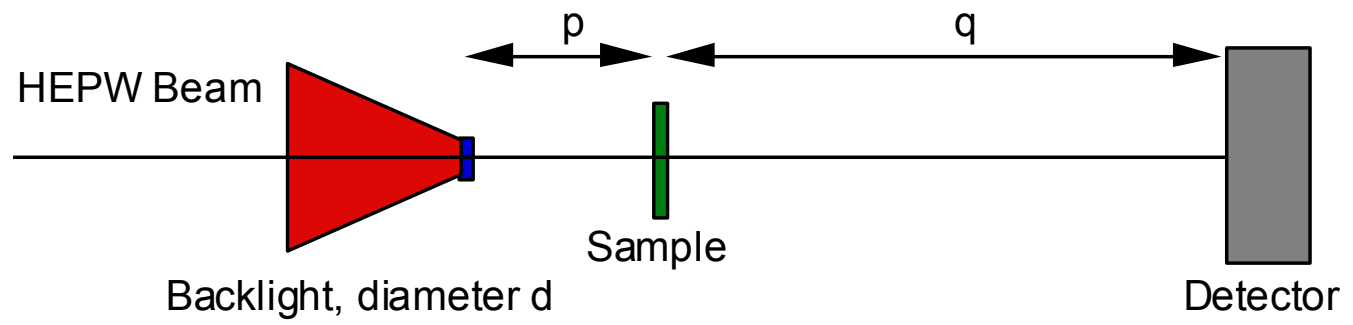

Referring to the sketch above, the number of photons detected on the projection plane in a point backlight scheme is given by:

$N\left(\frac{\gamma}{\text { res. element }}\right)=F\left(\frac{\gamma}{\text { sphere *area }}\right) * \frac{\pi d^{2}}{4} * \frac{\pi \sigma^{2}}{4 * 4 \pi p^{2}} * T($ transmission $) * \varepsilon($ detection efficiency $)$

$$
=F \frac{\pi d^{2}}{4} \frac{\sigma^{2}}{16 p^{2}} T \varepsilon
$$

The source diameter $\mathrm{d}$ required for a spatial resolution $\sigma$ is:

$$
d=\frac{(p+q)}{q} \sigma=\frac{M}{M-1} \sigma
$$


where $\mathrm{q}=(\mathrm{M}-1) \mathrm{p}$, where $\mathrm{M}$ is the magnification (which will be determined by the minimum achievable detector spatial resolution). $\mathrm{N}$ can then be written as:

$$
N=F \frac{\pi(p+q)^{2}}{p^{2} q^{2}} \frac{\sigma^{4}}{64} T \varepsilon=F \frac{\pi M^{2}}{(M-1)^{2} p^{2}} \frac{\sigma^{4}}{64} T \varepsilon
$$

Assume the nominal thickness of the foil is $\mathrm{x}$, and the transmission of the foil is $\exp (-\mu \mathrm{x})$. Then, for two adjacent regions differing by thickness 1 ,

$$
\begin{gathered}
N_{1}=F \varepsilon \frac{\pi M^{2} \sigma^{4}}{64(M-1)^{2} p^{2}} \mathrm{e}^{-\mu \mathrm{x}} \\
N_{2}=F \varepsilon \frac{\pi M^{2} \sigma^{4}}{64(M-1)^{2} p^{2}} \mathrm{e}^{-\mu(\mathrm{x}+1)}=N_{1} \mathrm{e}^{-\mu l}
\end{gathered}
$$

We wish to measure 1 from the ratio $\mathrm{N}_{1} / \mathrm{N}_{2}$,

$$
l=\frac{1}{\mu} \ln \left(\frac{N_{1}}{N_{2}}\right)
$$

The uncertainty in 1 is given by standard error propagation methods as:

$$
\begin{gathered}
\Delta l=\sqrt{\left(\frac{d l}{d N_{1}} \Delta N_{1}\right)^{2}+\left(\frac{d l}{d N_{2}} \Delta N_{2}\right)^{2}} \\
=\frac{1}{\mu} \sqrt{\left(\frac{\Delta N_{1}}{N_{1}}\right)^{2}+\left(\frac{\Delta N_{2}}{N_{2}}\right)^{2}} \\
\text { but } \Delta N=\sqrt{N}, \text { so } \\
\Delta l=\frac{1}{\mu} \sqrt{\frac{N_{1}+N_{2}}{N_{1} N_{2}}}
\end{gathered}
$$

So that if we wish to measure 1 to $10 \% \mathrm{~S} / \mathrm{N}$, we need:

$$
\frac{l}{\Delta l}=\frac{\ln \left(N_{1} / N_{2}\right)}{\sqrt{\frac{N_{1}+N_{2}}{N_{1} N_{2}}}}=10
$$

Inverting this and solving for $\mathrm{N}_{1}$, we have:

$$
N_{1}=\frac{100}{(\mu l)^{2}}\left(1+e^{\mu l}\right)=\frac{1 e 4}{(\mu x)^{2}}\left(1+e^{\mu \mathrm{x} / 10}\right)
$$


Using the explicit form of $\mathrm{N}_{1}$ for point-projection found above, we then can find the minimum source brightness that will be sufficient for this experiment:

$$
F>2.037 e 5 \frac{(M-1)^{2} p^{2}}{(\mu x)^{2} \varepsilon M^{2} \sigma^{4}} e^{\mu x}\left(1+e^{\mu x / 10}\right)
$$

This source brightness is minimized by a specific choice of $\mu \mathrm{x}$ which is close to $2(\mu \mathrm{x}=1.896)$, for which the number of photons/resolution element required is 6144. Substituting $\mu \mathrm{x}=1.896$ and $\sigma=10 \mu \mathrm{m}$, we have:

$$
F>8.335 e 13 \frac{(M-1)^{2} p^{2}}{\varepsilon M^{2}}
$$

By suitable choice of parameters $\mathrm{M}$, $\mathrm{p}$ and $\varepsilon$, we need to meet the $1 \mathrm{~mm}$ field-of-view requirement while having $\mathrm{F}$ less than or equal to the expected source brightness. If we assume that we can produce a $60 \mu \mathrm{m}$-diameter $\mathrm{Sm}$ K-alpha source (typical of existing data) with a conversion efficiency of $10^{-4} \mathrm{~J} / \mathrm{J} *$ sphere (an optimistic estimate on the high end of existing data), then a $1 \mathrm{~kJ}$ NIF HEPW beam would produce a source brightness of:

$$
\mathrm{F}_{\text {expected }}=5.52 \mathrm{e} 15 \text { (photons } / \mathrm{mm}^{2} * \text { sphere) }
$$

(a) Variation \#1a: Use low-magnification $(\mathrm{M}<2)$ point-projection imaging with a single multilayer mirror having arbitrary lateral and depth grading.

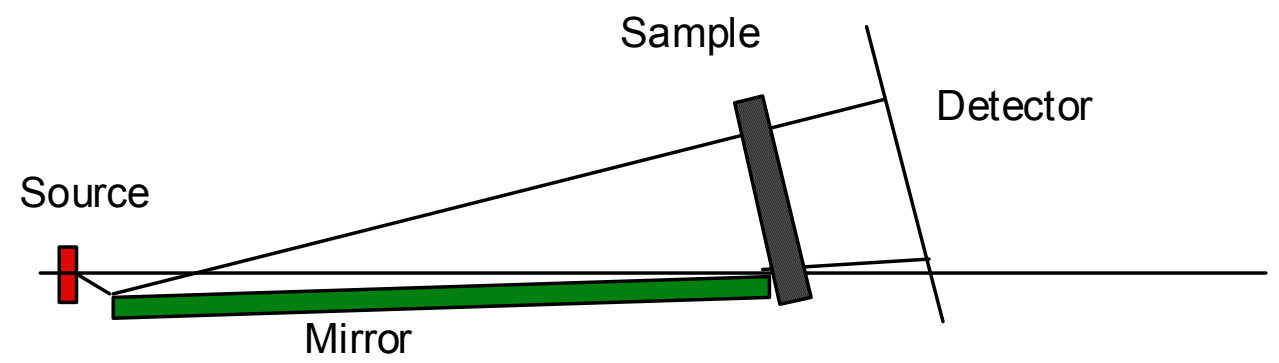

If we monochromatize the source with a flat multilayer mirror, the minimum value of $\mathrm{p}$ will be determined by the required field of view and the minimum achievable $2 \mathrm{~d}$ spacing of the mirror. In this variation, the mirror would be between the source and the sample, and the detector would be placed close behind the sample.

Mirrors with minimum $2 \mathrm{~d}=35$ angstroms are achievable, giving a Bragg angle at $40 \mathrm{keV}$ of $\sim 0.5$ degrees. If we assume the mirror coatings are arbitrarily gradeable laterally and in depth (subject to the minimum $2 \mathrm{~d}=35$ angstroms), and that the mirror fills the entire source/sample distance as shown above, then the maximum angular range subtended by the mirror is also $\sim 0.5$ degrees. A $1 \mathrm{~mm}$ sample subtends 0.5 degrees at a distance $\mathrm{p}=115 \mathrm{~mm}$, which is therefore the minimum source/sample distance and the approximate length of the mirror.

A $60 \mu \mathrm{m}$-diameter source will provide $10 \mu \mathrm{m}$ spatial resolution for magnifications less than 1.2X, but there is no advantage in going below $\mathrm{M}=1.2$ since the useful source diameter is larger than the actual source, and expanding the laser beam to fill this larger area would drop brightness proportionally. With $\mathrm{M}=1.2$ and $\sigma=10 \mu \mathrm{m}$, we would need a detector capable of $12 \mu \mathrm{m}$ spatial resolution at $40 \mathrm{keV}$. 
A CsI scintillator $12 \mu \mathrm{m}$ thick (assumed necessary to achieve $12 \mu \mathrm{m}$ spatial resolution) would have an energy absorption efficiency of 5\% at $40 \mathrm{keV}$ (NIST data tables). Behind the scintillator, we would need to use a microscope objective to re-image the $1.2 \mathrm{~mm}$ field of view onto a CCD camera. Assuming the maximum field of view is limited by the numerical aperture, the highest-magnification objective we could use would be $16 \mathrm{X}$ with a numerical aperture of 0.32 (Melles Griot catalog). A $1.2 \mathrm{~mm}$ field would then be magnified to $\sim 20 \mathrm{~mm}$ at the CCD, which is reasonable. As long as many scintillation photons per detected x-ray are collected by the lens and detected at the CCD, the numerical aperture does not effect the x-ray photon detection efficiency $\varepsilon$ since it does not degrade the photon statistics.

So, with $\mathrm{p}=115 \mathrm{~mm}, \mathrm{M}=1.2 \mathrm{X}, \varepsilon=5 \%$, and assuming the mirror reflectivity is an optimistic $90 \%$, we have the requirement:

$$
\mathrm{F}>6.8 \mathrm{e} 17 \text { (photons } / \mathrm{mm}^{2} * \text { sphere) }
$$

This implies that Variation 1a will not work, since the required source brightness is more than two orders of magnitude $(\sim 120 \mathrm{X})$ larger than the expected source brightness.

\section{Solutions for Variation \#1a:}

Reduce the field of view requirement? If we reduce the field of view requirement we can move the source closer to the sample. Keeping the same magnification $\mathrm{M}=1.2 \mathrm{X}$ and assuming the same detection efficiency, we would need to move the source in to a distance $p=10.3 \mathrm{~mm}$, giving a detector/source distance of $2.1 \mathrm{~mm}$ and a field of view of $90 \mu \mathrm{m}$ in the meridional direction. This seems plausible, though mounting and aligning the $10 \mathrm{~mm}$-long mirror between the source and sample would be a challenge, and might be further considered if $\mathrm{a} \sim 100 \mu \mathrm{m}$ field is acceptable.

Relax the $\mathbf{S} / \mathbf{N}$ requirement? The required source brightness scales as the desired S/N squared, but making up a factor of 100 in photon deficit is too large since it would violate the assumption that $\Delta \mathrm{l}$ is less than 1.

Improve the detection efficiency? A dramatic improvement in detection efficiency would solve most of the photometrics problem, since the estimated efficiency is only $5 \%$. If a $100 \%$-efficient detector were found with $12 \mu \mathrm{m}$ spatial resolution at $40 \mathrm{keV}$, then the photon deficit would be reduced to a factor of $\sim 6$. Some relaxation in the field of view requirement and the $\mathrm{S} / \mathrm{N}$ requirement might then make up the remaining difference.

\section{Other issues with Variation \#1a:}

- Damage to the mirror during the experiment, possibly before the short-pulse x-rays arrive.

- Damage to the detector due to very close proximity to the laser-irradiated sample.

- Mirror fabrication. The assumptions above were that a mirror can be made with a peak reflectivity of $90 \%$ and a lateral grading of the $2 \mathrm{~d}$ spacing ranging from $\sim 35 \AA$ to $\sim 2000 \AA$ along the $115 \mathrm{~mm}$-long mirror (assuming the near end of the mirror is $2 \mathrm{~mm}$ from the source and the Bragg angle varies between 0.5 and 0.0087 degrees). This seems unlikely, and any reduction in the achievable variation in $2 \mathrm{~d}$ spacing across the mirror will further restrict the field of view. The lateral grading problem could become less severe in the reduced FOV case where $\mathrm{p}=10.3 \mathrm{~mm}$ (varying from $\sim 35 \AA$ to $\sim 180 \AA$, with Bragg angles between 0.5 and 0.097 degrees), but the variation would now occur over a $10 \mathrm{~mm}$ length of mirror instead of $115 \mathrm{~mm}$.

A much more severe mirror fabrication issue results from the need to depth-grade the mirror coating in order to cover the range of angles subtended by the $60 \mu \mathrm{m}$ source. At $2 \mathrm{~mm}, 60 \mu \mathrm{m}$ subtends 1.7 degrees, and at $100 \mathrm{~mm}$ it subtends 0.03 degrees; in both extremes this variation is larger than the central Bragg angle, so that the entire $60 \mu \mathrm{m}$ source cannot be utilized. A depth grading sufficient to 
cover the fraction of the source visible would essentially be a broadband coating, and even if one could be made with good reflectivity the benefit of monochromatizing the source would be lost.

Overall practicality: Variation \#1a does not appear to be a viable solution. The photometrics do not look promising unless a "miracle detector" can be found with near-100\% efficiency at $40 \mathrm{keV}$ and $\sim 10$ $\mu \mathrm{m}$ spatial resolution. Even if one could be obtained, issues with mirror and detector damage and especially mirror fabrication would remain. Without a significantly improved detector, a reduced field of view $(\sim 90 \mu \mathrm{m})$ would be necessary in order to meet the source brightness requirements, and this in turn would introduce other practical problems without solving the mirror and detector damage and mirror fabrication issues. Essentially, for this problem the drawbacks of using a low-magnification geometry outweigh the benefits when using a flat multilayer mirror for monochromatization.

(b) Variation \#1b: Use high-magnification $(\mathrm{M}>>1)$ point-projection imaging with a single multilayer mirror having arbitrary lateral and depth grading.

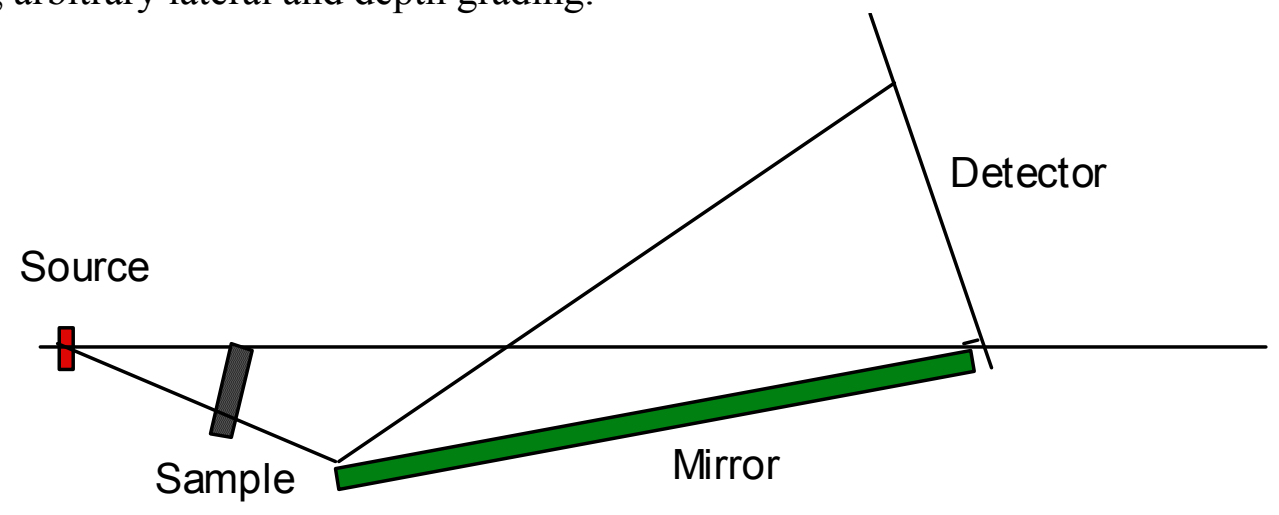

Here we move to high-magnification imaging by moving the detector far from the sample, and by placing the mirror between the sample and the detector. If we place the front of the mirror as close as possible to the sample, and for $M>>1$, the solid angle subtended by the mirror could also approach 0.5 degrees, as above. The source/sample distance is unchanged at $\mathrm{p}=115 \mathrm{~mm}$.

For $\mathrm{M}>>1$, the $60 \mu \mathrm{m}$ source is too large, and we would need to aperture it down to $\sim 10 \mu \mathrm{m}$, or alternatively use a more complicated target like a cone-focus and wire. We assume for now that either of these can be done with no loss in source brightness (photons/unit area).

One advantage of this scheme is that a more efficient, lower-resolution detector might be used. If we assume $\mathrm{M}=15 \mathrm{X}$, then a detector with $\sim 150 \mu \mathrm{m}$ detector resolution could be used, and a CsI scintillator this thick could have a detection efficiency of 50\%. Hardware issues with the mirror would also be simplified, and damage to the detector would be less of a concern.

So, with $\mathrm{p}=115 \mathrm{~mm}, \mathrm{M}=15 \mathrm{X}, \varepsilon=50 \%$, and assuming the mirror reflectivity is $90 \%$, we have the requirement: 
$\mathrm{F}>2.1 \mathrm{e} 18$ (photons $/ \mathrm{mm}^{2} *$ sphere)

This implies that Variation $1 \mathrm{~b}$ will not work either, since the required source brightness is a factor of $380 \mathrm{X}$ larger than the expected source brightness. This is because losses in (M-1)/M more than make up for gains due to improved detection efficiency.

\section{Solutions for Variation \#1b:}

Reduce the field of view requirement? Keeping the same magnification $\mathrm{M}=15 \mathrm{X}$ and assuming the same detection efficiency, we would need to move the source in to a distance $p=5.9 \mathrm{~mm}$, giving a detector/source distance of $82.6 \mathrm{~mm}$ and a field of view of $50 \mu \mathrm{m}$ in the meridional direction. This seems plausible if $\mathrm{a} \sim 50 \mu \mathrm{m}$ field is acceptable.

Relax the $\mathbf{S} / \mathbf{N}$ requirement? This will not help, for reasons discussed in connection with Variation 1a.

Improve the detection efficiency? This could only gain a factor of 2, so there are no significant improvements possible here.

\section{Other issues with Variation \#1b:}

- Damage to the mirror during the experiment, possibly before the short-pulse x-rays arrive. The problem is little better than the case of Variation 1a, though perhaps more of the mirror would remain undamaged.

- Mirror fabrication. The same problems discussed in the case of Variation 1a remain here, but they are generally less severe. For the $\mathrm{p}=115 \mathrm{~mm}$ case, assuming the mirror fills the region between the sample and the detector except for a $2 \mathrm{~mm}$ gap at the sample, the range of Bragg angles would be 0.5 to 0.034 degrees, the range of $2 \mathrm{~d}$ spacings would be $35 \AA$ to $522 \AA$ across the $1600 \mathrm{~mm}$-long mirror, and a graded coating with $\Delta \lambda / \lambda \sim 200$ would be sufficient to allow the full $10 \mu \mathrm{m}$ source to be utilized. For the $\mathrm{p}=5.9 \mathrm{~mm}$ case, the range of Bragg angles would be 0.5 to 0.045 degrees, and a graded coating with $\Delta \lambda / \lambda \sim 15$ would be sufficient to allow the full $10 \mu \mathrm{m}$ source to be utilized. Neither set of mirror parameters seems especially challenging.

- Source size reduction. A $10 \mu \mathrm{m}$ source with the same brightness as a $60 \mu \mathrm{m}$ foil source would need to be made. Aperturing the source with a pinhole is possible, but a Ta substrate would need to be $240 \mu \mathrm{m}$ thick in order to achieve a background level equal to the signal level (due to substrate transmission at $40 \mathrm{keV}$ ), and a thickness of $550 \mu \mathrm{m}$ in order to achieve a signal/background ratio of 10 . This is comparable to the 0.5 -degree mirror acceptance angle, so the pinhole would need to be carefully aligned. A cone-focus wire target is another possibility, but the actual brightness of this type of target remains to be quantified.

Overall practicality: While the high-magnification geometry appears to minimize most of the mirror fabrication problems, the photometric deficit is much larger with no apparent solution. Variation \#1b appears to be a viable option only if a significantly reduced field of view $(\sim 50 \mu \mathrm{m})$ is acceptable. Possible issues still remain with mirror damage and source size reduction.

(c) Variation \#1c: Use high-mag $(\mathrm{M}>>1)$ point-projection imaging with an array of flat multilayer mirrors having arbitrary lateral and depth grading, in order to make up the field of view lost due to the need to reduce the source/sample distance in order to achieve the required photometrics specifications.

One can imagine stacking mirrors in such a way to increase the collection angle of an array of mirrors, and thereby increase the field of view. 


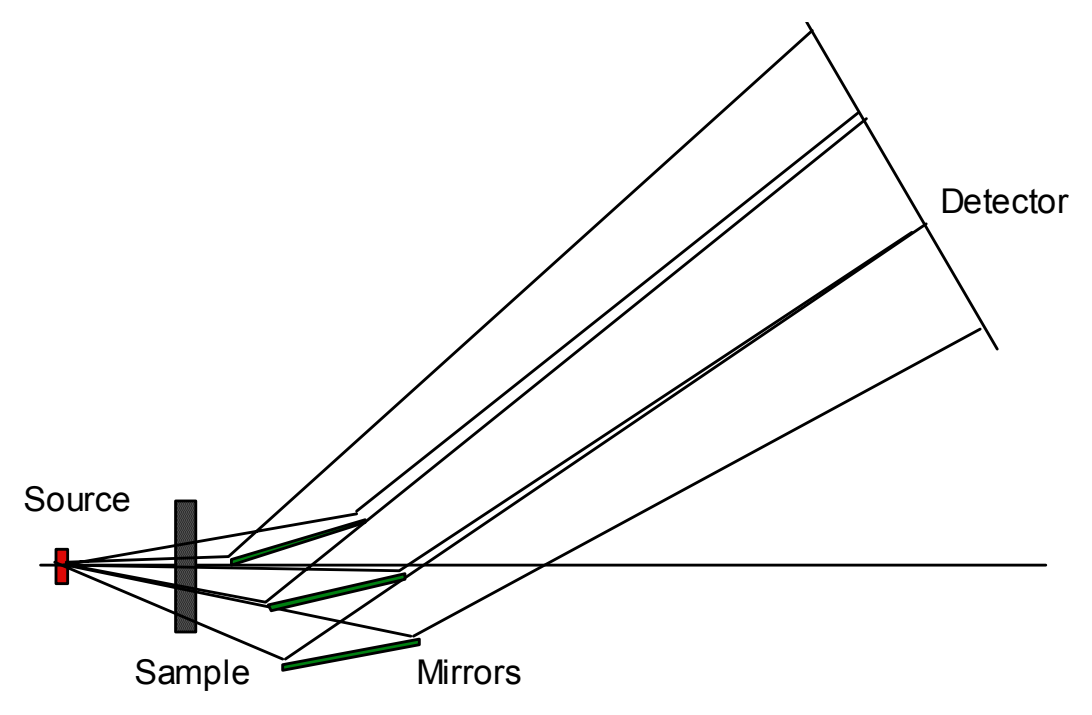

However in order for this geometry to provide full coverage of the field of view, the front end of each mirror surface would need to fall along the line between the source and the back end of the mirror below it. Typical superpolished multilayer mirror substrates are $0.5 "$ thick, compared with the $70 \mu \mathrm{m}$ a single $\sim 80 \mathrm{~mm}$-long mirror subtends in the $\mathrm{p}=5.9 \mathrm{~mm}$ geometry. Unless substrates $<<70 \mu \mathrm{m}$ thick can be obtained with adequate surface figure and roughness specifications, there will unavoidably be large gaps in the field of view. This seems unacceptable unless the gaps are small, but it appears instead that the gaps will be much larger than the field of view covered by each mirror.

We can also imagine linking several mirrors end-to-end (rather than stacking them). However in this case, the need for each mirror to avoid blocking the field of view of the mirror in front of it, and the fixed maximum Bragg angle for each mirror, force the sequence of mirrors towards a cylindrical surface with the source on the Rowland circle. In this geometry the fields overlap.

\section{Solutions for Variation \#1c:}

Use thin-foil mirrors? If very thin $(\sim 10 \mu \mathrm{m})$ mirror substrates were used, then gaps in the field of view in the stacked-mirror geometry would be minimized. It seems unlikely that such foil mirrors would have adequate surface properties, however.

\section{Other issues with Variation \#1c:}

- In addition to the issues raised with regard to Variation $1 \mathrm{~b}$, mounting and aligning multiple mirrors would be challenging.

Overall practicality: There do not appear to be multiple flat mirror solutions which meet the experimental requirements without either leaving large gaps in the field of view, or overlapping multiple fields of view onto the detector. Extremely thin $(\sim 10 \mu \mathrm{m})$ foil mirrors could allow most of the field of view to be observed with minimal gaps, but it seems unlikely that these would have adequate surface properties. If I am missing some clever solution to these problems then perhaps this geometry might be reconsidered, but otherwise it appears to create more problems than it solves.

II. Concept \#2. Use point-projection backlighting in a very high-magnification geometry, without a multilayer mirror monochromatizer, and use single-pixel selection to obtain a quasi-monochromatic radiograph. 
If the detector is moved back to a great distance, and is sufficient to allow a magnified resolution element to cover at least as many pixels as the number of photons/resolution element required, then single-hit spectroscopy techniques could be used to eliminate photons at energies outside the desired bandwidth.

For this experiment, approximately 6100 photons/resolution element must be detected in order to meet the $\mathrm{S} / \mathrm{N}$ requirement. Detecting these photons individually would require a $\sim 78 \times 78$ array of pixels for each magnified resolution element, so that an individual pixel would cover a distance equivalent to $\sim 0.128 \mu \mathrm{m}$ at the sample. For a typical $\sim 100 \mu \mathrm{m}$ pixel width, this would mean the point-projection magnification would be $780 \mathrm{X}$, and the detector would need to be a $780 \mathrm{~mm}$-square array of $100 \mu \mathrm{m}$ pixels. For a source/sample distance of $5.9 \mathrm{~mm}$, this requires placing the detector at a distance of 4.6 meters. These are best-case numbers, where $40 \mathrm{keV}$ photons dominate the spectrum; in actuality we would probably need several times as many pixels/resolution element in order to allow the $\mathrm{S} / \mathrm{N}$ to be achieved with quasi-monochromatic $\mathrm{x}$-rays alone (after elimination of high-energy $\mathrm{X}$-rays, in particular).

\section{Solutions for Concept \#2:}

Very large mosaic CdTe array? It is conceivable that a very large pixelated array could be made by arranging many chips in a mosaic pattern. Alternatively a CsI scintillator array could in principle be made arbitrarily large, though relaying the large image to an optical CCD would be challenging.

\section{Other issues with Concept \#2:}

- Attenuation along the flight path and through vacuum windows.

- Backgrounds from scattered and fluorescent $\mathrm{x}$-rays.

- Reduction of the source size to $\sim 10 \mu \mathrm{m}$, perhaps with a pinhole.

Overall practicality: This geometry could be interesting if a very large pixelated array could be found and could be placed at large ( $\sim 10$ meter) distances from the target along a non-attenuating line of sight. In the absence of such a large array, however, it is not practical.

III. Concept \#3. Use a bent-crystal or bent-multilayer mirror microscope of the Kirkpatrick-Baez design, with the source on the Rowland circle.

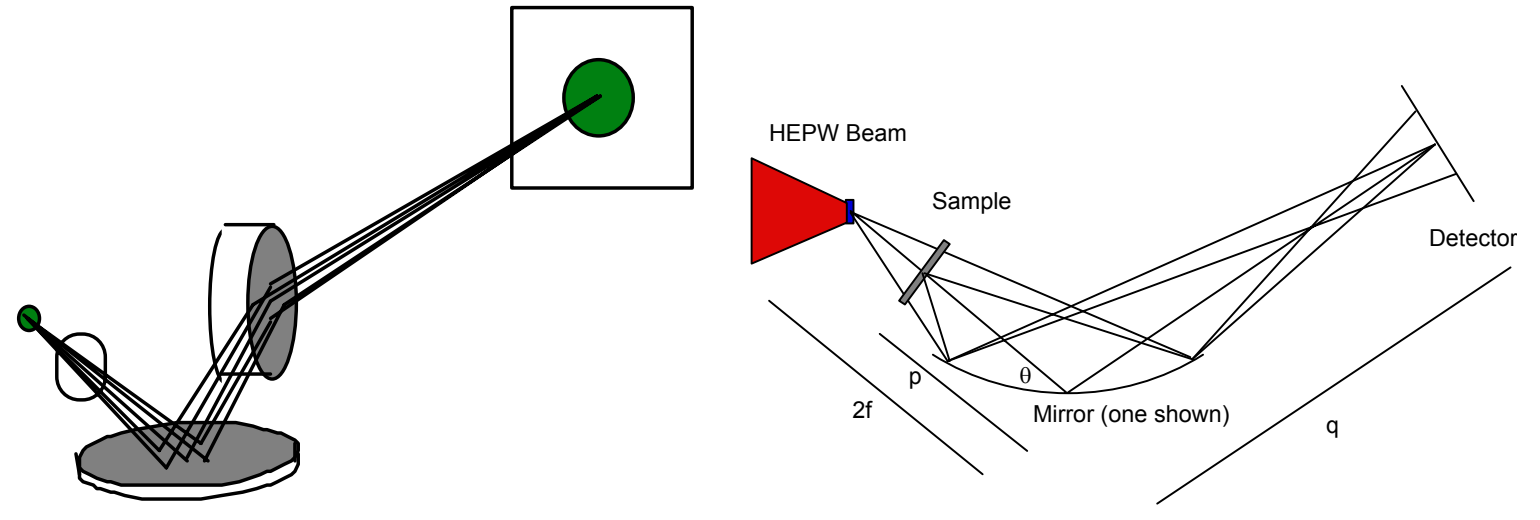


In this geometry, placing the source on the Rowland circle gives a constant Bragg angle across the mirror surface, which at small Bragg angles is necessary in order to allow a reasonable source size to be utilized (useful field $\sim 2 p \tan (\theta) \mathrm{B}$, where $\mathrm{p}$ is the source distance and $\mathrm{B}$ is either the relative line bandwidth $\Delta \lambda / \lambda$ or the mirror bandwidth, whichever is smaller).

In reality we cannot place both mirrors on the Rowland circle because they must be at physically different locations, and so the Bragg angle cannot be constant across both mirrors. Complications also arise using a spherical mirror rather than an idealized cylindrical mirror, but we can ignore these details for the time being and revisit them if the scheme looks promising.

From earlier work (J. A. Koch et al., Appl. Opt. 37, 1784 (1998)), we can write the number of photons/unit area at the image of the source as:

$$
e=F \frac{\Omega \eta}{4 \pi} T
$$

where $\mathrm{F}$ is the source brightness as above (photons/sphere/unit area), $\Omega$ is the optic collection solid angle, $\eta$ is the efficiency through the solid angle, and $\mathrm{T}$ is the sample transmission. Moving away from this focus towards the image of the sample, the focused beam will diverge according to the solid angle. For sample magnification $\mathrm{M}$ and imaging focal length $\mathrm{f}$, the area of this defocused source is:

$$
A=\frac{\pi}{4}(M-1)^{2} f^{2} \Omega
$$

The number of photons/unit area in the sample image plane is equal to e times the ratio of this area to the area of the backlight source d, so:

$$
e_{\text {image }}=F \frac{\eta T}{4 \pi} \frac{d^{2}}{(M-1)^{2} f^{2}}
$$

and therefore the number of detected photons/resolution element is:

$$
N=F \eta T \varepsilon \frac{\sigma^{2} d^{2} M^{2}}{16(M-1)^{2} f^{2}}
$$

Equating this to the necessary number of photons/resolution element found above,

$$
N_{1}=\frac{1 e 4}{(\mu x)^{2}}\left(1+e^{\mu x / 10}\right)
$$

we can solve for $\mathrm{F}$ as before, using $\mathrm{d}=60 \mu \mathrm{m}, \mathrm{s}=10 \mu \mathrm{m}$, and $\mu \mathrm{x}=1.896$ :

$$
F>1.818 e 12 \frac{f^{2}(M-1)^{2}}{\eta \varepsilon M^{2}}
$$

We find that the solid angle of the microscope does not enter into the calculation of the minimum source brightness, though it does enter into the calculation of the field of view below. 
At grazing angles of incidence the principle geometrical optics aberration will be obliquity of field. From earlier work (J. A. Koch et al., Appl. Opt. 37, 1784 (1998)) we can calculate the maximum opening angle $\phi$ that will give a resolution $\sigma$ over a field of view L for a Rowland circle backlight, and this is

$$
\phi=\frac{\sigma}{L} \tan \theta
$$

This is equal to the angle subtended by the sample as viewed from the source, which is $L /(2 \mathrm{f}-\mathrm{p})=\mathrm{ML} / \mathrm{f}(\mathrm{M}-1)$. This puts a minimum limit on the optic focal length $f$, which is:

$$
f=\frac{M L^{2}}{\sigma(M-1) \tan \theta}
$$

Substituting into the equation for the minimum source brightness, we have:

$$
F>1.818 e 12 \frac{L^{4}}{\eta \varepsilon \sigma^{2} \tan ^{2} \theta}
$$

For $\sigma=10 \mu \mathrm{m}, \mathrm{L}=1 \mathrm{~mm}$, and $\varepsilon=50 \%$, this is:

$$
F>\frac{3.64 e 16}{\eta \tan ^{2} \theta}
$$

Now we can consider two cases, a multilayer mirror KB and a bent-crystal KB.

From earlier work (J. A. Koch et al., Appl. Opt. 37, 1784 (1998)), the efficiency of a multilayer-mirror KB (with a mirror bandwidth much broader than the source bandwidth) operating with a Rowland circle backlight source is simply equal to the peak reflectivity squared. If the optimistic $90 \%$ value is used, and with $\theta=0.5$ degrees, we have:

$$
F>5.9 e 20 \text { (photons } / \mathrm{mm}^{2} * \text { sphere) }
$$

This implies that this system will not work, since the required brightness is 5 orders of magnitude larger than the expected brightness.

For a crystal KB, the Rowland-circle backlight efficiency can be written as

$$
\eta=\frac{B}{(\Delta \lambda / \lambda)}
$$

where $\mathrm{B}$ is the relative crystal bandwidth $\Delta \mathrm{E} / \mathrm{E}$. Considering the specific example of quartz $5052(2 \mathrm{~d}=1.624 \AA$, one of the smallest of practical crystals, for which $\theta=10.97$ degrees) assuming $B$ for this crystal is comparable to that expected for other quartz crystal planes $(1 / 30,000)$, and assuming $\Delta \lambda / \lambda$ scales with energy from calculations for Ti K-alpha $(\Delta \lambda / \lambda \sim 1.8 \mathrm{e}-3)$, we have:

$$
F>5.23 e 19 \text { (photons } / \mathrm{mm}^{2} * \text { sphere) }
$$

This implies that this system will not work either, since the required brightness is 4 orders of magnitude larger than the expected brightness. 


\section{Solutions for Concept \#3:}

None are obvious.

Overall practicality: The strong scaling of the required brightness with field of view (fourth power) makes a Rowland-circle Kirkpatrick Baez design impractical for this experiment. A bent-crystal design appears to be more attractive because the Bragg angle is much larger than can be obtained with a multilayer mirror, but a field of view of $\sim 100 \mu \mathrm{m}$ would be the limit over which $10 \mu \mathrm{m}$ spatial resolution could be achieved. For a multilayer-mirror KB, the field of view would be restricted to $\sim 55 \mu \mathrm{m}$. Both these reduced field-of-view cases would only achieve useful brightness using very short focal-length optics, for which the details of the optical design would be important to investigate before the concept is pursued any further. This concept might be more attractive for other experiments which do not require a large field of view but do require very high spatial resolution, since $\sim \mu \mathrm{m}$-level resolution is achievable with this type of microscope.

IV. Concept \#4. A spherical crystal imager in a Rowland circle backlight geometry.

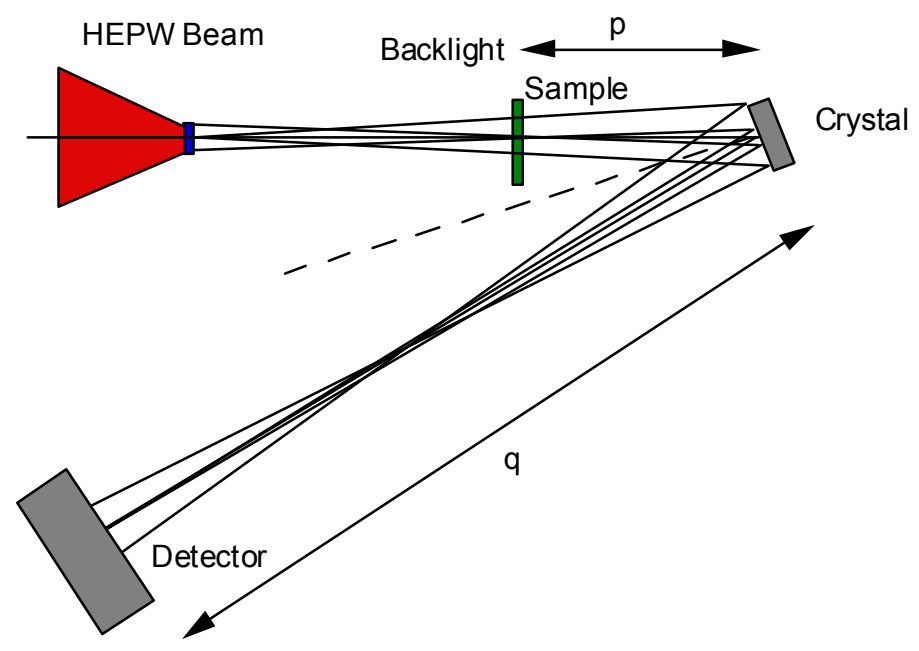

In this geometry, we can make use of most of the derivations from Concept \#3, down to:

$$
F>1.818 e 12 \frac{f^{2}(M-1)^{2}}{\eta \varepsilon M^{2}}
$$

For a near-normal incidence crystal, mm-scale fields of view are easily achieved, and the limitation on the focal length $\mathrm{f}$ comes instead from practical fabrication limits. Assuming a bend radius of $100 \mathrm{~mm}$ is achievable (this is certainly practical with mica), then $f=50 \mathrm{~mm}$. Assuming $M=15$ and $\varepsilon=50 \%$, we have:

$$
F>\frac{7.92 e 15}{\eta} \text { (photons } / \mathrm{mm}^{2} * \text { sphere) }
$$

We can see immediately that this geometry will not meet the requirements either, even if an extremely efficient crystal could be found, since even a $100 \%$-efficient crystal fails to meet the brightness requirement by a factor of 1.4. Actual crystal efficiencies would probably be many orders of magnitude less than this, since a crystal would need to be operated in high order if it is to reflect $40 \mathrm{keV}$ x-rays at near normal incidence. 


\section{Solutions for Concept \#4:}

None are obvious.

Overall practicality: It appears that this concept is not practical, because a crystal with realistic efficiency would probably fail to meet the photometric requirements by orders of magnitude.

V. Concept \#5. Use a bent-multilayer mirror microscope of the Kirkpatrick-Baez design, with an area backlight.

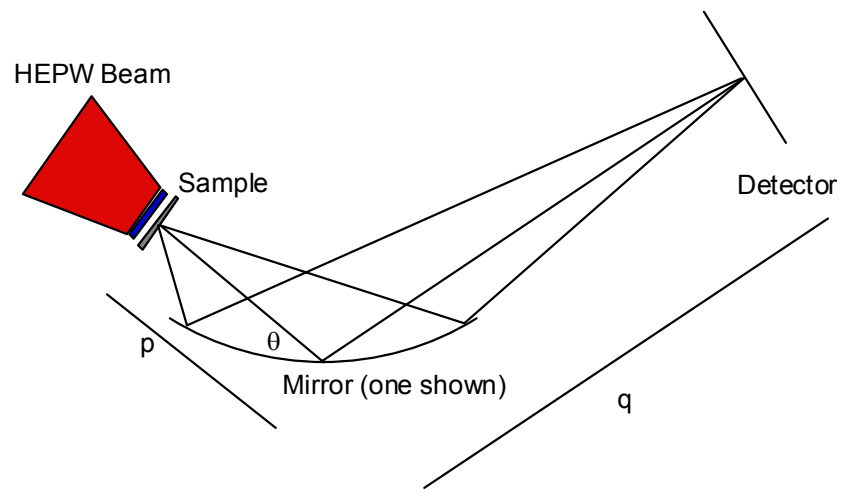

Here, we use an area backlight instead of a point-like backlight on the Rowland circle, and place the backlight just behind the sample. In this geometry the Bragg angle varies significantly across the surface, so we only consider a bent multilayer mirror version (crystal bandwidths are too small).

We can write the number of photons/resolution element at the image of the sample as above:

$$
N=F \frac{\Omega \eta \varepsilon \sigma^{2}}{16} T=\frac{1 e 4}{(\mu x)^{2}} e^{\mu x}\left(1+e^{\mu x / 10}\right)
$$

Assuming $\varepsilon=50 \%$ and $\sigma=0.01 \mathrm{~mm}$, we have:

$$
F>\frac{1.31 e 10}{\Omega \eta}
$$

Assuming again that obliquity of field is the dominant aberration limiting off-axis performance,

$$
\Omega=\left(\frac{L \sin \theta}{p}\right)^{2}=\left(\frac{2 M \sigma \tan \theta}{L(M+1)}\right)^{2}
$$

If we also assume that the efficiency is an optimistic $(90 \%)^{2}$, for $\mathrm{M}=15$ and $\theta=0.5$ degrees we have:

$$
F>6.0 e 17 \text { (photons } / \mathrm{mm}^{2} * \text { sphere) }
$$

However in order to create a $1 \mathrm{~mm}$ backlight we will need to defocus the laser. Assuming the laser energy and conversion efficiency are constant, we will then loose a factor of $(0.06)^{2}$ in source brightness, giving:

$$
F_{\text {expected }}=2.0 e 13 \text { (photons } / \mathrm{mm}^{2} * \text { sphere) }
$$


We find again that the KB design cannot meet the photometrics requirements, by more than 4 orders of magnitude.

Solutions for Concept \#5:

None are obvious.

Overall practicality: There is little advantage to using an area backlight for a KB design in this application, and it fails to meet the requirements.

VI. Concept \#6. Use a spherical crystal with an area backlight.

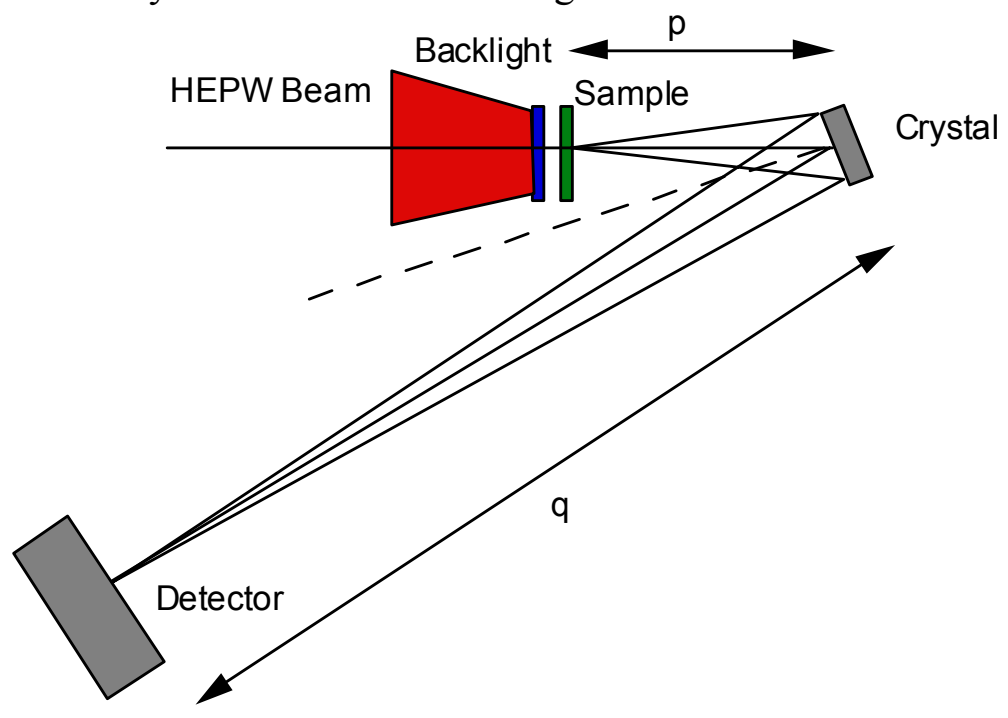

Making use of the results from Concept \#5, we can again write:

$$
F>\frac{1.31 e 10}{\Omega \eta}
$$

For the spherical crystal, assuming the useful area is limited by astigmatism rather than by the line width (generally true unless the Bragg angle is within $\sim 2$ degrees of normal incidence), we can write:

$$
\Omega=\left(\frac{L}{p}\right)^{2} \sin \theta=\left(\frac{M \sigma}{p(M+1)(1-\sin \theta)}\right)^{2} \sin \theta
$$

In this case the efficiency is simply:

$$
\eta=\frac{B}{(\Delta \lambda / \lambda)}
$$

Assuming $\mathrm{p}=50 \mathrm{~mm}$ (for a $100 \mathrm{~mm}$ crystal radius), $\mathrm{M}=15$ and $\Delta \lambda / \lambda \sim 1.8 \mathrm{e}-3$, we have: 


$$
F>\frac{6.71 e 14}{B \sin \theta}(1-\sin \theta)^{2}
$$

For $\theta$ near 90 degrees, we can write $\theta=\pi / 2-\delta$, and approximate the result as:

$$
F>\frac{1.68 e 14}{B} \delta^{4}
$$

Choosing $\delta=2$ degrees and $\mathrm{B}=1 / 30,000$ (typical of quartz in first order), we would then have

$$
F>7.5 e 12 \text { (photons } / \mathrm{mm}^{2} * \text { sphere) }
$$

This compares with an expected $1 \mathrm{~mm}$-backlight brightness from above of:

$$
F_{\text {expected }}=2.0 e 13 \text { (photons } / \mathrm{mm}^{2} * \text { sphere }
$$

We find that this concept would indeed provide adequate image brightness, provided a crystal could be found to reflect $40 \mathrm{keV}$ x-rays within 2 degrees of normal incidence and with a bandwidth of 1/30,000.

\section{Other issues with Concept \#6:}

- None are obvious, beyond practical issues of mounting and aligning a crystal.

Overall practicality: This concept would appear to be a viable option, but only if a spherically-bent crystal could be found to operate very near normal incidence at $40 \mathrm{keV}$ with good efficiency, necessarily in a high Bragg order. Further investigation should be pursued to determine if such a crystal exists, though it would appear highly unlikely since high-order Bragg reflections are generally weak (mica is a notable exception).

VII. Concept \#7. Use a Wolter microscope with an area backlight. 


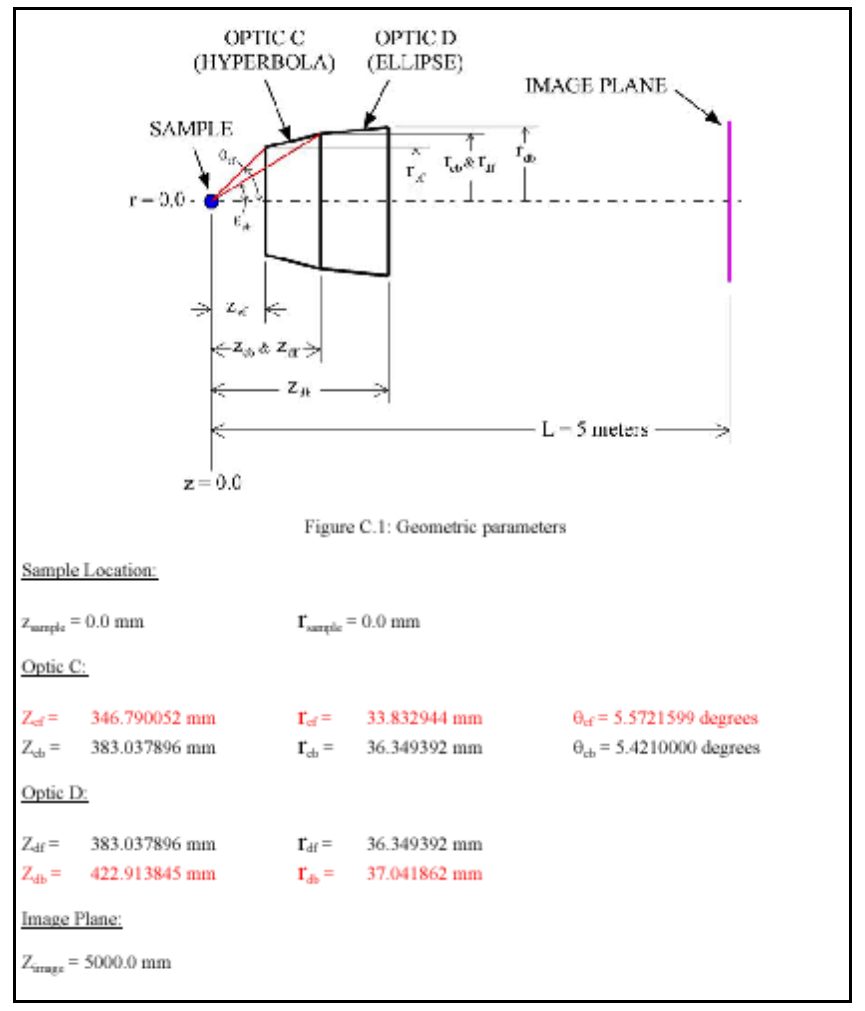

Rather than attempt to design a $40 \mathrm{keV}$ Wolter microscope on paper, I instead use an existing documented design (W. Nederbragt, UCRL-ID-150702, sketched above) of an $8 \mathrm{keV}$ microscope as a starting point. This design, if it could be fabricated, would have sub-10- $\mu \mathrm{m}$ spatial resolution throughout a $\sim 1 \mathrm{~mm}$ field of view, and would use depth- and laterally-graded mirror surfaces.

Making use again of the results from Concept \#5, we can write:

$$
F>\frac{1.31 e 10}{\Omega \eta}
$$

Here, the collection solid angle is the annular region shown above. The solid angle is approximately $1.59 \mathrm{e}-3 \mathrm{Sr}$. The efficiency through this collection angle would depend on the details of the multilayer mirror, but if we assume that there are no bandwidth complications and an optimistic peak reflectivity of $90 \%$, we would then have:

$$
F>1.0 e 13\left(\text { photons } / \mathrm{mm}^{2} * \text { sphere }\right)
$$

However, since the Nederbragt design operates with central Bragg angles near 1.5 degrees (Appendix D of the design report), it would not work at $40 \mathrm{keV}$ x-ray energies.

We can estimate the loss in collection angle at $40 \mathrm{keV}$ by assuming that the useful mirror lengths do not change, and that the meridional angle subtended by the first mirror therefore simply scales with the central Bragg angle. This is precisely the case for a $\mathrm{KB}$ microscope, and is a reasonable assumption for the present purposes.

The total collection solid angle is then reduced by a factor of $3^{2}=9$, and the estimated required source brightness becomes: 


$$
F>9.0 e 13 \text { (photons } / \mathrm{mm}^{2} * \text { sphere) }
$$

This compares with an expected $1 \mathrm{~mm}$-backlight brightness from above of:

$$
F_{\text {expected }}=2.0 e 13 \text { (photons } / \mathrm{mm}^{2} * \text { sphere) }
$$

This result implies that a $40 \mathrm{keV}$ Wolter could possibly meet both the source brightness and the field of view requirements, within factors of a few. Any photometric deficit could probably be made up by changing the design parameters, for example increasing the collection solid angle at the expense of on-axis spatial resolution (which in the design above is far better than the current requirement of $10 \mu \mathrm{m}$ ).

\section{Other issues with Concept \#7:}

- Mirror fabrication. This is the central problem with this type of microscope. Fabrication of an optic with adequate surface figure and roughness is a significant technical challenge that must be solved even before the multilayer mirror challenges can be addressed. I cannot say whether or not an adequate-on-paper design could ever actually be made, or at what cost, but recent LLNL experience trying to build the optic for the Nederbragt microscope by replication techniques (Harry Martz, "Advancing the Technology R\&D of Tabletop Mesoscale Nondestructive Characterization, 2003 Engineering Strategic Initiative) showed that the problems are formidable and expensive. Funding for the Martz SI was stopped after the first optic mandrel failed to meet it's technical requirements because there did not appear to be a clear path forward.

- Mirror damage. Given the large costs involved with building such a microscope, great care would be required to ensure that the optics were not damaged during the experiments.

Overall practicality: On paper this approach looks promising, but a successful implementation would require a detailed design study of the type performed by Nederbragt, and especially some new ideas on how to fabricate adequate mirrors. If the mirror fabrication problem could be solved, then the multilayer coating problems would probably not be insurmountable. Pursuing this approach would probably require a significant laboratory commitment at least on the level of the Martz SI.

VIII. Concept \#8. Use a refractive bubble lens and an area backlight.

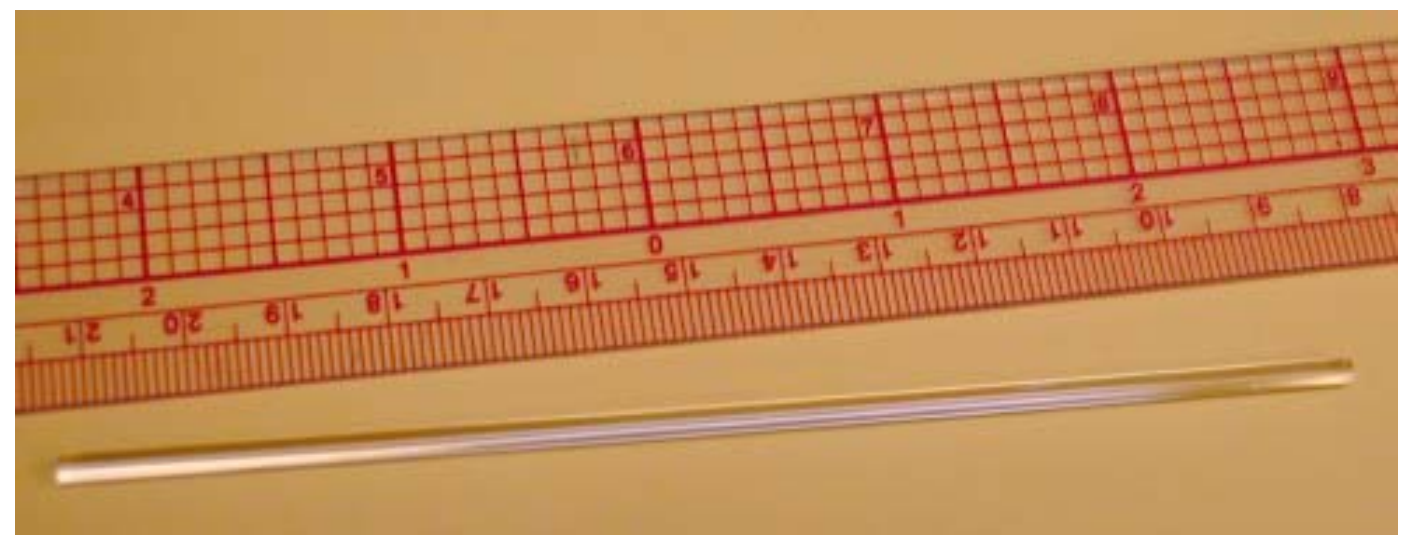

We currently have a compound polymer bubble lens manufactured by Adelphi Technologies, that was intended for use at $22 \mathrm{keV}$. This lens has not yet been tested, but the manufacturer claims it should be capable of $\sim 1 \mu \mathrm{m}$ spatial resolution over a $\sim 2 \mathrm{~mm}$ field of view, operating with a $200 \mu \mathrm{m}$ full lens aperture and with a focal length of $300 \mathrm{~mm}$. The manufacturer stated that the lens focal length should scale approximately as $\mathrm{E}^{2}$, with 
little change in the other parameters, so we would expect a $990 \mathrm{~mm}$ focal length and a field of view proportionally larger. The $22 \mathrm{keV}$ lens has a transmission of $23 \%$.

We can estimate the required source brightness using the same results from Concept \#5:

$$
F>\frac{1.31 e 10}{\Omega \eta}
$$

Here, the collection solid angle of a $200 \mu \mathrm{m}$ aperture at a distance of $1056 \mathrm{~mm}$ (for $\mathrm{M}=15 \mathrm{imaging}$ ) is $2.8 \mathrm{e}-8$ $\mathrm{Sr}$, and the efficiency through that solid angle is 0.23 , so the required source brightness is:

$$
F>2.0 e 18 \text { (photons } / \mathrm{mm}^{2} * \text { sphere) }
$$

This compares with an expected $1 \mathrm{~mm}$-backlight brightness from above of:

$$
F_{\text {expected }}=2.0 e 13 \text { (photons } / \mathrm{mm}^{2} * \text { sphere) }
$$

The required source brightness is 5 orders of magnitude larger than the expected source brightness, indicating that this concept will not work.

\section{Solutions for Concept \#8:}

Array of lenses? In the same way we can improve pinhole imaging data quality by using an array of pinholes, we might imagine making an array of bubble lenses and adding the images together to improve the signal/noise ratio. However making up five orders of magnitude in source brightness would require an array of this many bubble lenses, which would be hundreds per side. Such an array could conceivably be made, though aligning the individual elements would be a challenge. Additionally, the individual images would be so weak that detector noise limitations would need to be taken into account.

Reduce the field of view? If we give up on a $1 \mathrm{~mm}$ field of view and keep a best-focus laser beam with a $60 \mu \mathrm{m}$ backlight, the photometric deficit drops to only a factor of $\sim 360$. A 20x20 array of bubble lenses could then quite plausibly be made to eliminate the remaining deficit.

\section{Other issues with Concept \#8:}

- Very distant detector on a long line of sight.

- Chromatic aberrations from other x-rays. Other x-ray energies would be present apart from 40 $\mathrm{keV}$, and these would be focused at different distances, adding background noise and degrading the image spatial resolution. A detailed design would need to address these effects.

Overall practicality: This approach has some advantages over complex mirror-based microscopes, and could potentially work if a very large array of bubble lenses were created; however, the complexities of such an array, including data analysis, seem formidable for a full $1 \mathrm{~mm}$ field of view. This approach seems better suited to very high-resolution imaging of static samples at lower x-ray energies, where photons can be collected over long periods of time. It might also be suitable for a relaxed field-of-view experiment, particularly if very high spatial resolution is desired.

IX. Concept \#9. Use a Laue crystal imager and an area backlight. 
This is essentially a speculative possibility, based on the results of Faenov et al. (Rev. Sci. Instruments 74, 2224 (2003)). That paper described a spherically-bent transmission Laue crystal that was used to form $M=2$ magnified x-ray images of a grid using a $22 \mathrm{keV}$ microfocus x-ray source. The data showed $<10 \mu \mathrm{m}$ spatial resolution over $\mathrm{a} \sim 1.5 \mathrm{~mm}$ field of view. The image formation mechanism is not obvious, and was left unexplained in the paper, but the data clearly show an image. The overall efficiency of this approach is also unclear, and cannot easily be estimated from the paper, but a good exposure took 4-5 hours on DEF film. The advantages and disadvantages of this approach are therefore difficult to state, but further research in this area might prove fruitful.

X. Concept \#10. Use a Fresnel zone plate and an area backlight.

Using the results from Concept $\# 5$, assuming $\varepsilon=50 \%$ and $\sigma=0.01 \mathrm{~mm}$, for an area backlight imaging geometry we require the source brightness $\mathrm{F}$ to be

$$
F>\frac{1.31 e 10}{\Omega \eta}
$$

We can look at existing commercially-available zone-plate characteristics to estimate what performance might be obtained for this experiment. Xradia, Inc., sells zone plates optimized for a variety of applications and x-ray energies, and their web site (http://www.xradia.com/zpl_pd.htm) list their properties. The focal length obtained is:

$$
f=\frac{D(\Delta R)}{\lambda}
$$

where $\mathrm{D}$ is the diameter of the zone plate, $\Delta \mathrm{R}$ is the width of the outermost zone, and $\lambda$ is the $\mathrm{x}$-ray wavelength. They do not sell zone plates intended for $40 \mathrm{keV}$ x-rays, but we can estimate an upper performance bound by using their numbers for $9 \mathrm{keV}$ zone plates. The three zone plates listed (ZP100-160-16, ZP100-320-16 and $\mathrm{ZP} 100-4000-16$ ) have the same $\Delta \mathrm{R}=0.1 \mu \mathrm{m}$ and the same focusing efficiency $\eta=31 \%$, and have varying diameters of $160 \mu \mathrm{m}, 320 \mu \mathrm{m}$ and $4 \mathrm{~mm}$. The focal lengths for $\lambda=1.38 \AA$ x-rays are then $116 \mathrm{~mm}, 232 \mathrm{~mm}$ and $2904 \mathrm{~mm}$.

Assuming we will operate at high magnification, where the zone plate/sample distance is essentially equal to the focal length, then the solid angle subtended by all three zone plates is the same,

$$
\Omega=\frac{\pi(0.08)^{2}}{(116)^{2}}=\frac{\pi(0.16)^{2}}{(232)^{2}}=\frac{\pi(4)^{2}}{(2904)^{2}}=1.49 e-6
$$

With $\eta=0.31$, we have:

$$
F>2.8 e 16 \text { (photons } / \mathrm{mm}^{2} * \text { sphere) }
$$

Again, in order to create a $1 \mathrm{~mm}$ backlight we will need to defocus the laser, and the result from Concept \#5 was:

$$
F_{\text {expected }}=2.0 e 13 \text { (photons } / \mathrm{mm}^{2} * \text { sphere) }
$$


The required source brightness is 3 orders of magnitude larger than the expected source brightness, indicating that this concept will not work.

\section{Solutions for Concept \#10:}

Use multilayer mirror-technology zone plates? The collection solid angle scales as $(\lambda / \Delta R)^{2}$, so going from $9 \mathrm{keV}$ to $40 \mathrm{keV}$ and recovering the factor of 1400 photon deficit would require $\Delta \mathrm{R}=5 \AA$, assuming the same $31 \%$ efficiency. This is less than multilayer mirror fabrication technology limits, but only by a factor of a few. A more serious problem would be the field-of-view limitation, since a $5 \AA$ outer zone width would appear rapidly larger for off-axis object points and the efficiency would rapidly fall.

\section{Other issues with Concept \#10:}

- Damage to the expensive zone plate.

- Chromatic aberrations from other x-rays. Other x-ray energies would be present and would be focused at different distances (Xradia's $9 \mathrm{keV}$ zone plates have $>10 \%$ efficiency for x-ray energies between 5 and $24 \mathrm{keV}$ ), adding background noise and degrading the image spatial resolution. A detailed design would need to address these effects.

Overall practicality: This approach does not appear to offer any advantages for this experiment, and is better suited for applications requiring extremely high spatial-resolution over small fields of view. A jelly-roll type zone plate could in principle offer higher efficiency, but probably with a corresponding reduction in useful field-of-view.

X. Concept \#10. Use a Bragg-Fresnel structure with an area backlight.

There has been some theoretical and experimental work involving one-dimensional phase zone-plate structures etched or machined into flat or cylindricaly bent crystals. The advantage of this geometry is the ease of spectral tuning by changing the Bragg angle and the crystal bend radius, and the elimination of x-ray energies outside the narrow crystal bandpass. However, given the poor performance of bent crystal imagers and twodimensional zone plates uncovered above, it seems highly unlikely that this type of imager would meet the photometric requirements for the experiment investigated here.

\section{Summary}

For this particular two-dimensional radiography experiment, there are no clear paths for meeting the experimental requirements using quasi-monochromatic x-rays. Though the analysis above is based on foil source characteristics that might be improved upon by development of a higher-brightness small source, the photometric deficits are still too large $\left(>>(60 / 10)^{2}\right.$, the largest plausible increase in source brightness obtainable with a cone-focus wire target) to allow the experimental requirements to be met. In short, this is a challenging experiment even with the relatively high brightness achievable with short-pulse high-intensity laser backlights.

\section{Possible monochromatic imaging solutions include:}

1) Aperturing the expected $\sim 60 \mu \mathrm{m}$ source to $\sim 10 \mu \mathrm{m}$ (or developing a restricted-dimension $\sim 10 \mu \mathrm{m}$ source) and detecting x-rays on a very large detector for single-hit spectroscopic point-projection imaging. This would require detector development, but seems plausible in principle. Identification of suitable detector materials would need to be made in order to determine if this option is really practical. 
2) Development of a spherically-bent imaging crystal with high efficiency in high Bragg orders, using it in an area-backlight imaging geometry. This solution would only be plausible if such a crystal could be identified, and would require fabrication and testing of a variety of crystals bent to spherical shapes.

3) Development of a high-efficiency small source (for example with a cone-focus wire), relaxing the signal-tonoise requirements by a factor of three, and using point-projection imaging geometry with a flat graded multilayer mirror. A $10 \mu \mathrm{m}$ source with the same conversion efficiency as the $60 \mu \mathrm{m}$ foil source would improve the brightness by a factor of 36 , and the factor of three relaxation in signal-to-noise would then permit the point-projection Concept $\# 1 \mathrm{~b}$ to meet the photometric requirements. This solution could be promising if source development and optimization efforts are successful.

4) Relaxation of other experimental requirements, particularly field of view, so that some of the other options explored above might become practical. The field-of-view requirement would realistically need to be reduced to $\sim 100 \mu \mathrm{m}$ in order for these other concepts to become possible solutions.

Failing these, the experimental requirements can only be met using a broad-band x-ray backlight, with spectral characteristics determined by the backlight material, laser intensity, filtration and detector sensitivity curve.

\section{For this case, plausible broadband imaging options include:}

1) Using the expected full $\sim 60 \mu \mathrm{m}$ source dimension from a foil backlight, and using a low-magnification contact-radiography geometry with a high-resolution scintillator detector. This is Concept \#1a, without the multilayer mirror. This would require detector development, but seems quite plausible in principle. Xradia has done extensive development of high-efficiency high-resolution scintillators, and we could potentially team with them or simply purchase scintillators from them. Provided the emission spectrum can be suitably characterized during the experiment, this appears to be the most promising overall option. Other issues to investigate include damage to the scintillator during the experiment, and possible debris and shrapnel effects on laser optics and other diagnostic hardware in the target chamber.

2) Reducing the source dimension by physically restricting the source, and using high-magnification pointprojection imaging. This would require source optimization, particularly using a cone-focus wire source, and would only be practical if $\mathrm{x}$-ray emission from the cone is insignificant compared with $\mathrm{x}$-ray emission from the wire. Alternatively, a backlit pinhole could be used provided it is sufficiently opaque to all x-rays in the detected energy bandwidth of the detector.

3) Pinhole imaging with an area backlight. Neutron pinhole imaging has been performed and is planned for the NIF, and it is possible that a suitably designed pinhole could work for this experiment as well. However, given the numbers in Concept \#1, a pinhole would need to subtend a solid angle of 6.6e-4 $\mathrm{Sr}$, which for a $10 \mu \mathrm{m}$ pinhole requires a distance of $\sim 1 \mathrm{~mm}$ from the sample, in order to meet the photometric requirements. This would also raise issues of field-of-view.

This work was performed under the auspices of the U.S. Department of Energy by the University of California, Lawrence Livermore National Laboratory under Contract No. W-7405-Eng-48. 